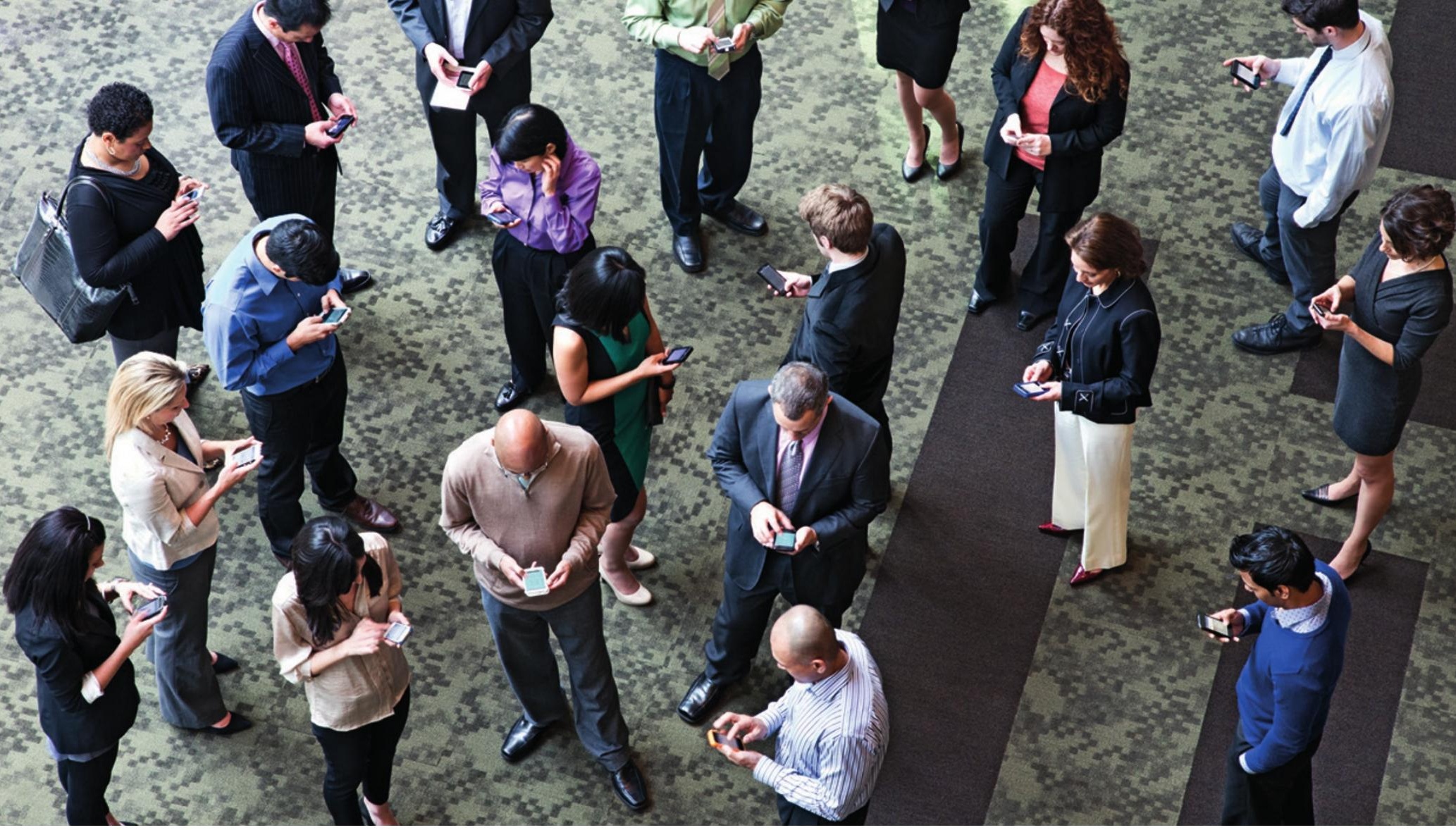

Smartphones are ubiquitous; they owe most of this success to the lithium-ion batteries that power them.

LITHIUM BATTERIES

\title{
To the limits of lithium
}

\section{Researchers are developing a type of battery that has ten times the power of conventionalbatteries.}

\section{BY ERIC C. EVARTS}

$\mathrm{T}$ There is one major reason why you are able to carry around a powerful microcomputer in your pocket. Lithium-ion batteries have been credited for revolutionizing communications and transportation, enabling the rise of super-slim smartphones and electric cars with a practical range.

These innovations were possible because lithium-ion batteries can be much smaller and lighter than the previous generation of nickelcadmium batteries, but still provide the same power. Better still, lithium-ion batteries retain their charge for longer and are composed of much less toxic materials.

As the lightest metal on the periodic table, and the one most eager to shed its electrons, lithium is the ideal element to make powerful, portable batteries. It can do the most work with the least mass and the fewest chemical complications.

But the development of lithium batteries was fraught with difficulties. The first versions - developed by the Texas-based oil company Exxon in response to the energy shortages during the 1970s oil crisis - were not rechargeable and used lithium compounds that created toxic by-products in the electrolyte, unlike later lithium-ion batteries. They went on to power the first generation of digital watches, but early prototypes were ticking time bombs. Gases from the electrolyte could build up inside the battery and burst into flames as soon as they made contact with air.

Over the next 30 years or so, progress in lithium battery development had regular setbacks owing to battery fires and angry customers. Constant recalls gave lithium batteries a bad name, and sceptics assumed that they would never be safe enough for the mass market.

The promise of lithium batteries to transform the way society uses energy, however, is so powerful that it has created a gold rush among scientists, engineers, venture capitalists and entrepreneurs to tame their volatility.

"Modern society is completely dependent on fossil fuels, so there's a huge incentive to find a replacement for the internal combustion engine," says John Goodenough, a solid-state physicist at the University of Texas at Austin who is widely seen as the father of today's lithium-ion batteries. "We have to find a way to liberate society from that dependence. What we're talking about is to be able to get electric energy from sun and wind, instead of from coal. But that's not feasible unless you have storage."

Goodenough is credited with three of the four major breakthroughs that led to the widespread success of lithium-ion batteries. In the late 1970s, he developed cathodes containing lithium cobalt oxide, which still power most personal electronics today (K. Mizushima et al. Mater. Res. Bull. 15, 783-789; 1980). Later, he and a battery materials researcher, Michael Thackeray, followed this up with lithium manganese oxide cathodes, which power most electric cars today as well as some medical devices (M. Thackeray et al. Mater. Res. Bull. 18, 461$472 ; 1983)$. In the 1990s, Goodenough carried on working after his retirement and developed an even cheaper and more stable cathode made of lithium iron phosphate, which is now widely used in power tools.

These chemistries are safer than the first generation of lithium batteries because the electrodes contain no free lithium. Instead, a chemical latticework tightly binds lithium ions 


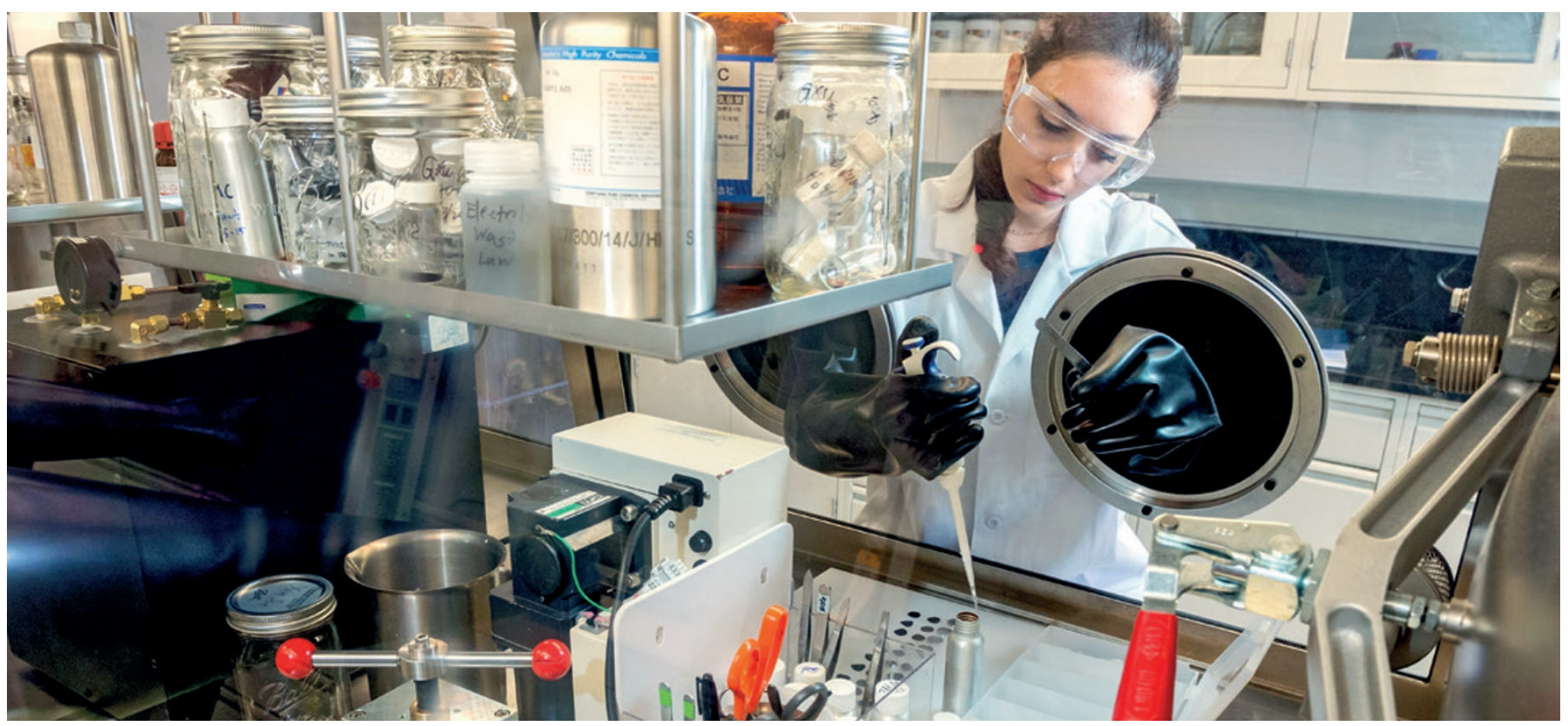

A researcher tests prototype electrodes as part of efforts to find ways of making batteries last longer and charge more quickly.

to complex metal oxide crystal structures in the positive cathode (where electrons and lithium ions travel as the battery is powering a device). The lithium ions move directly through the electrolyte and do not react with other elements.

\section{THE LITHIUM LANDSCAPE}

As far as lithium batteries have come, today's technology is still limited. Many mobile phones cannot make it through a day without being recharged. Most electric cars can travel for only 160 kilometres or less before they have to be plugged in for hours to recharge. The Tesla Model S offers a market-leading range of more than 300 kilometres, but at a cost: prices start at US\$71,000.

Battery scientists and engineers have been making batteries 5-10\% more efficient every year for the past 25 years, says George Crabtree, a materials scientist at Argonne National Laboratory in Illinois. But he worries that this pace of progress may be slowing. "Unless a brand new oxide is discovered, that's where we are," he says. "Where you are going to pull the next rabbit of out the hat, we're not sure." Even Goodenough plays down the impact that the

\section{"I don't think you can get a solid-state battery with long life."} technology has had. "We don't really have electric cars yet," he says. "You've got to lower the cost, make them safer and lengthen the driving range so you don't give people range anxiety."

The trick to developing batteries that last longer, charge more quickly and cost less is to pack as many lithium ions into each of the electrodes as possible, and get them to flow back and forth between the anode and cathode as quickly as possible, without letting them flow out of control.

For example, Goodenough's former partner,
Thackeray, who now works on electrochemical storage technologies at Argonne, developed a crystalline molecular structure known as a spinel to transport lithium ions safely into the cathode as the battery discharges. The ions can only flow through channels in the spinel, making it highly stable. The more tightly that scientists can control lithium ions in structures such as these, the more stable the battery becomes. The technology does have a significant drawback, however: the less freely that lithium ions can move, the less power that the battery has. And as the transition from nickel-cadmium to lithium-ion batteries proved, boosting power is the best way to reduce size, weight and cost.

But it may not be the only way. Ping Liu, who directs research into advanced materials for energy efficiency and storage at the Advanced Research Projects Agency-Energy (ARPA-E), part of the US Department of Energy in Washington DC, says that improving safety and stability can also make batteries smaller and less expensive. That is because such advances would allow engineers to eliminate the electronic controllers, armour plating, cell insulators and cooling systems that today's electric cars need to protect the batteries.

\section{POSITIVE AND NEGATIVE DEVELOPMENTS}

The most substantial progress so far has occurred at the cathode, the positive pole that absorbs lithium ions as the battery provides its power. Scientists have been working on making thinner layers of cathode material using nanomaterials, such as carbon, with the goal of speeding up the chemical flow of lithium through the battery by shortening the distance that ions have to travel. Some scientists are working on material as thin as a single atom. But efforts on this front have still not produced the advantages hoped for. "No one has managed to do it well," says Crabtree. "It's difficult to make the layers come out even at such a small scale."

The next breakthroughs are expected to come at the other end of the battery, from better anodes, Crabtree says. Anodes store the lithium ions when the battery is charged and send them to the cathode as the battery releases power. When the Japanese electronics giant Sony introduced carbon anodes to replace more troublesome lithium metal anodes in the early 1990 s, the batteries lost some power. Now engineers want to get it back.

One big problem with today's graphite anodes, and the lithium metal anodes that preceded them, is that the lithium ions returning to the anode when the battery is being charged do not coat the surface evenly. Instead, they grow like tree limbs in tiny crystalline structures called dendrites.

"Carbon can only accept lithium at a given rate," says materials scientist Nitash Balsara at the University of California, Berkeley. "If you try to send lithium [through the battery] too fast [while charging], the lithium doesn't really go into the graphite, it sticks on the outside. It becomes a safety hazard." And the smaller the battery, the easier it is for dendrites to grow all the way across the electrolyte and contact the opposite pole, shorting out the battery, Goodenough says.

Permeable membranes called separators are used to prevent contact between the electrodes, and thus stop short circuits, while allowing the flow of electrolyte. But dendrites can break off and block pores in separators, shortening the life of the battery.

\section{MORE POWER}

Anodes can be made from silicon, which can hold up to ten times as much lithium per gram as graphite and therefore generate more power. 
But silicon poses its own problem: it expands to more than three times its normal size when the battery is charged and the anode is filled with lithium ions. This swelling breaks down the electrical bonds in the anode and stops the battery from working. It can also break the adjacent parts of the battery, such as the separator and even the battery case, and thus cause a fire.

Yi Cui, a materials scientist at Stanford University, California, who has been developing lithium-ion batteries for 15 years, is one of the scientists working on thinner electrode materials. He is developing silicon nanowires that stick up from the anode like fibres from a carpet and do not break the electrical bonds when they swell. But he says that the technology is still five years from commercialization. He is also experimenting with ways to improve graphite anodes, using two-dimensional graphene to absorb lithium more quickly while charging. But he says that this work too has a long way to go.

The ideal would be to return to a pure lithium-metal anode. "Forget silicon, if you can go straight to lithium-metal, that is the end goal," says Liu.

Compared with graphite anodes, lithiummetal anodes can absorb ten times more lithium ions while charging - and without silicon's swelling problem. Such a battery could reach a key performance metric for use in electric vehicles: delivering the 300 watt-hours of energy per kilogram that is needed to enable an electric vehicle to go the same distance on one charge that a petroleum-powered car can on a full tank. But this milestone would require other safety advances, such as a solid electrolyte or better separators, to allow charging without dendrite growth.

"I just don't see lithium metal with a liquid electrolyte as a commercial possibility," says Balsara. "That looks like TNT to me."

\section{SOLID ELECTROLYTES}

Solid electrolytes could bring the biggest breakthroughs yet (see page S8.) "If you could get rid of a liquid electrolyte, then you can get rid of all the combustible elements," says Liu. That would address the primary safety concern of lithium batteries. Liu's engineers are even designing a battery so strong and safe that it could ultimately form part of a car's body and absorb the impact in a crash, he says.

But solid electrolytes bring trade-offs, too. "They don't conduct electricity nearly as well as liquid electrolytes, so charging time goes up, and power goes down," Balsara says. And Goodenough adds: "I don't think you can get a solid-state battery with long life."

Goodenough aims to bridge the gap by developing a partial-solid electrolyte using a solid material next to the anode and a liquid next to the cathode. "It will achieve the same benefits as a solid electrolyte with longer life," he says, "because the anode material won't break down."

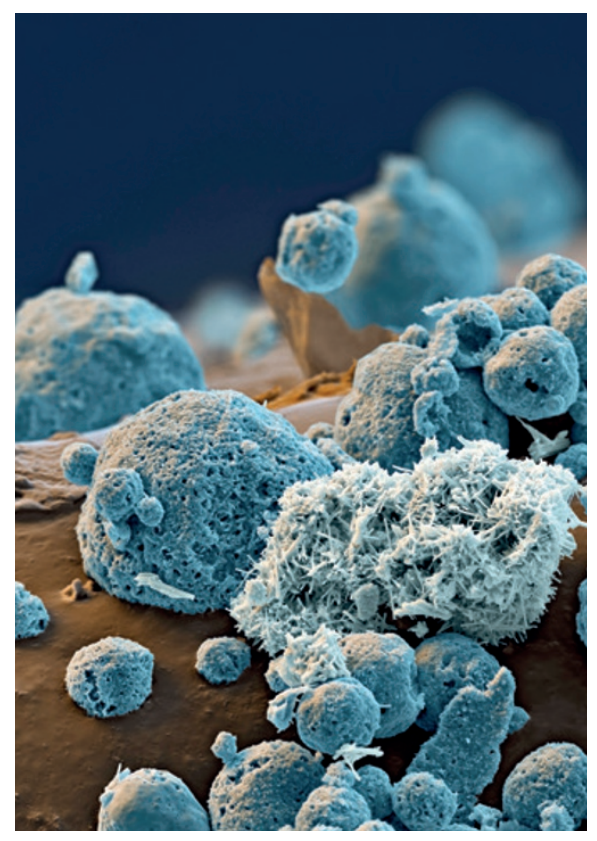

Lithium ion is used in rechargeable batteries.

He has built a test battery in his laboratory, but says he is still having problems with dendrite growth.

Liu says that the most promising developments are coming from scientists working on ceramic electrolytes. He points to an ARPA-Efunded project at the University of Maryland that has demonstrated a solid-state battery with a solid lithium anode that works using a ceramic electrolyte.

"Solid glass and ceramic electrolytes have significantly higher conductivity than plastic polymer," says Balsara, "and the conductivity is high enough that you might not have to sacrifice so much power."

Others working on new types of electrolytes for lithium-ion batteries include Yet-Ming Chiang, a materials scientist at the Massachusetts Institute of Technology in Cambridge, who helped to develop the lithium iron phosphate battery. He showed a new type of flow battery this summer that uses a semi-solid 'peanut butter-like' electrolyte. It solves the problem of how to get ions to flow quickly through a solid electrolyte by turning the battery design on its head, using liquid electrodes that are pumped over the semi-solid electrolyte. He says it could dramatically reduce the cost of producing lithium-ion batteries. Chiang has started a company called 24M, based in Cambridge, Massachusetts, to commercialize the invention, but it remains unproven.

\section{NOT JUST IONS}

As nanotechnology improves electrodes and electrolytes, the search for more power is bringing scientists full circle, back to batteries that use pure lithium rather than lithium ions. Lithium-sulfur and lithium-air batteries are not categorized as lithium-ion batteries because the lithium reacts in the electrolyte to form other compounds rather than simply flowing through the electrolyte and not reacting with it.

Lithium-sulfur batteries, similar to those batteries that Exxon experimented with in the 1970s, can store up to ten times the energy of a lithium-ion battery by weight. The problem is that the electrochemical reactions involved consume the sulfur and create other substances that dilute the electrolyte. Both processes prematurely kill the battery. "Ideally, you'd like an electrolyte that only transmits lithium," says Balsara. "Anything else it does is a problem."

To solve this complication, Cui, Crabtree and others are developing nanomaterials to encapsulate the sulfur. Cui says that his lab has demonstrated a lithium-sulfur battery that can last through 500 to 1,000 chargedischarge cycles. That could be enough for a mobile phone or laptop, he says. But its energy capacity is still too low, notes Cui, and a commercial prototype may be five years away.

Lithium-air batteries could turn out to be the ultimate lithium battery in terms of power, weight and cost. With a lithium-metal anode and a gaseous oxygen cathode, a lithium-air battery could store as much energy as a lithium-sulfur battery at even less cost, and potentially with less weight.

If the materials sound simple, however, the battery is not. For one thing, 'air' is a misnomer. The cathode has to be pure oxygen, without any of the moisture or carbon dioxide found in air. The systems to purify, pump and store the air add $30-70 \%$ to the battery's weight and size, says Crabtree. Although on paper lithium-air can deliver ten times the energy density of lithium-ion, "it will never achieve the factor of ten that you get from the back of the envelope", he says.

On top of that, the cathode oxidizes any organic electrolyte over time, diminishing the life of the battery.

Worst of all, because the reaction turns lithium into non-conductive dilithium oxide, the battery is difficult to recharge. Cui says that researchers have demonstrated a reasonable number of charges and discharges of lithium-air test batteries by encapsulating dilithium oxide on a conductive substrate, but he estimates that lithium-air batteries are at least ten years away from commercialization.

Their development may sound difficult, but better lithium batteries could deliver huge pay-offs. Beyond electric cars that could go 300 kilometres, they could provide the breakthrough that makes renewable energy such as solar and wind power ubiquitous, and finally break the developed world's dependence on fossil fuels.

Eric C. Evarts is a freelance writer in Ridgefield, Connecticut. 